Hans Bertram \& Carolin Deuflhard

\title{
Das einkommensabhängige Elterngeld als Element einer nachhaltigen Familienpolitik
}

\author{
The parental allowance as an element of a sustainable family policy
}

\begin{abstract}
Zusammenfassung:
Nachhaltige Familienpolitik erkennt die Leistungen der Familie für die Gesellschaft an und nimmt sie als Ausgangspunkt für die Unterstützung der Familie. Sie zeichnet sich durch klare Zielorientierungen und die Integration der verschiedenen familienpolitischen Leistungen aus. Vor diesem Hintergrund analysiert dieser Beitrag, ob und inwiefern aus der Entwicklung der Familienpolitik ein Paradigmenwechsel im Verständnis der familienpolitischen Kernelemente Infrastruktur, Zeit und Geld von einem Nachteils- zu einem Leistungsausgleich hervorgegangen ist. Denn erst auf dieser Grundlage konnte das Konzept nachhaltiger Familienpolitik formuliert werden. Anhand der Rekonstruktion der konzeptionellen Entwicklung und politischen Durchsetzung des einkommensabhängigen Elterngeldes seit den 1970er Jahren wird aufgezeigt, dass hier zum ersten Mal eine familienpolitische Maßnahme geschaffen wurde, die in dieses Konzept eingebettet ist. Die Analyse zeigt jedoch, dass das Elterngeld zwar ein Element einer nachhaltigen Familienpolitik ist, es aber bisher weder gelungen ist, eine am Gedanken des Leistungsausgleichs orientierte finanzielle Transferpolitik zu konzipieren noch eine Lebensverlaufsorientierung umzusetzen.
\end{abstract}

Schlagwörter: nachhaltige Familienpolitik, Leistungsausgleich, Kindertagesbetreuung, Lebensverlauf, Vereinbarkeit von Beruf und Familie, Erziehung, Sozialisation, Kinder- und Jugendhilfegesetz, Grundsicherung

\begin{abstract}
:
A sustainable family policy acknowledges the contributions of the family to society and takes it as point of departure for the support of the family. It is characterized by a clear goal orientation and the integration of different policy measures into a comprehensive strategy. Based on this concept, the article reflects the historical evolution of family policy focusing on the question whether and to what extent a paradigm shift from compensation to active support has occurred in the interpretation of policies in the fields of infrastructure, time and money. This is essential for the historical genesis of the parental allowance, considering that the emergence of the concept of a sustainable family policy was based on this paradigm shift. By means of reconstructing the conceptual evolution and political implementation of the parental allowance since the 1970s, we argue that this political measure is the first to be integrated in such a concept. The analysis shows that even though the parental allowance is a progressive element of a sustainable family policy, the implementation of a comprehensive strategy still lacks a shift towards the life course perspective and an independent basic income for children.
\end{abstract}

Key words: sustainability, family policy, childcare, life course, reconcilability of work and family, upbringing, socialization, basic income 


\section{Mutterschaftsurlaub, Erziehungsurlaub, Elterngeld und Elternzeit}

2007 trat das Gesetz zum einkommensabhängigen Elterngeld in Kraft, das von vielen als ein neues Element in der finanziellen Förderung von Familien und Kindern interpretiert wurde. Dabei steht das einkommensabhängige Elterngeld in einer langen Tradition politischer Maßnahmen, seit die sozialliberale Koalition 1979 den schon damals geltenden Mutterschutz um vier Monate Mutterschaftsurlaub ergänzte, damit die berufstätigen Mütter bis zum 6. Lebensmonat ihres Kindes zu Hause bleiben konnten. Diese Zeit war finanziell abgesichert und mit dem Recht auf den Wiedereinstieg in den Beruf verbunden (Hermann 1984). Diese Regelung wurde 1986 zum zehnmonatigen Erziehungsurlaub erweitert, den nun auch Mutter oder Vater nehmen konnten; auch diese zehn Monate waren finanziell abgesichert und mit dem Rückkehrrecht in den Beruf verknüpft. 1992 wurde der Erziehungsurlaub auf drei Jahre ausgeweitet; dabei wurde das Erziehungsgeld für zwei Jahre gezahlt, aber der Rechtsanspruch auf den Wiedereinstieg bestand drei Jahre lang. 2001 wurde der Erziehungsurlaub in Erziehungszeit umbenannt und die Möglichkeit geschaffen, ein Jahr der Erziehungszeit bis zum 8. Lebensjahr des Kindes zu nehmen. Zudem gab es nun einen Anspruch auf vollzeitnahe Teilzeit (bis zu 30 Stunden).

Aus dieser eher stichwortartigen Wiedergabe der Entwicklung wird deutlich, dass sowohl die zeitliche Freistellung für die Fürsorge für Kinder zusammen mit dem Anspruch, wieder in den Beruf zurückzukehren, keine neue Regelung ist, sondern bereits seit 30 Jahren besteht. Die ökonomische Sicherung dieser Zeit war, wenn auch bescheiden, vorgesehen und zudem wurden auch zunehmend die Möglichkeiten erweitert, um auch die Väter an der Erziehungszeit zu beteiligen. Die Begründungen des Wissenschaftlichen Beirats für Familienfragen (1984) oder die politischen Begründungen der verschiedenen Minister und Ministerinnen lassen eigentlich nur feststellen, dass das einkommensabhängige Elterngeld kein neues Konzept ist, sondern in seiner Grundkonfiguration seit Mitte der 1970er Jahre bekannt ist. ${ }^{1}$ Abbildung 1 zeigt, dass die finanziellen und zeitrechtlichen Regelungen des heutigen einkommensabhängigen Elterngeldes in der historischen Kontinuität früherer politischer Regelungsentscheidungen steht: Es wird deutlich, dass einerseits in der Bundesrepublik Deutschland seit den 1970er Jahren eine rege Debatte zu Fragen der Vereinbarkeit von Familie und Beruf stattfand und andererseits intensiv diskutiert wurde, wie die zunehmende Qualifikation junger Frauen auch außerhalb der Familie in der Arbeitswelt genutzt werden könnte (Erler/Jaeckel/Sass 1983).

1 Neu am einkommensabhängigen Elterngeld ist das explizite Lohnersatzelement. Allerdings wurde bereits Anfang der 1980er Jahre vor der Einführung des Bundeserziehungsgeldes darüber diskutiert, es als Lohnersatz auszuzahlen. Die Berechnungsgrundlage beim Bundeserziehungsgeld orientierte sich am durchschnittlichen Einkommen bei Vollerwerbstätigkeit und entsprach etwa der Hälfte eines solchen Einkommens (600 DM). Auch die Inanspruchnahme des Erziehungsurlaubs durch den Vater mit Arbeitsplatzgarantie war bereits seit 1986 möglich (Erler/Jaeckel/Sass 1983; Wissenschaftlicher Beirat für Familienfragen 1984). 
Abbildung 1: Entwicklungsstufen des einkommensabhängigen Elterngelds

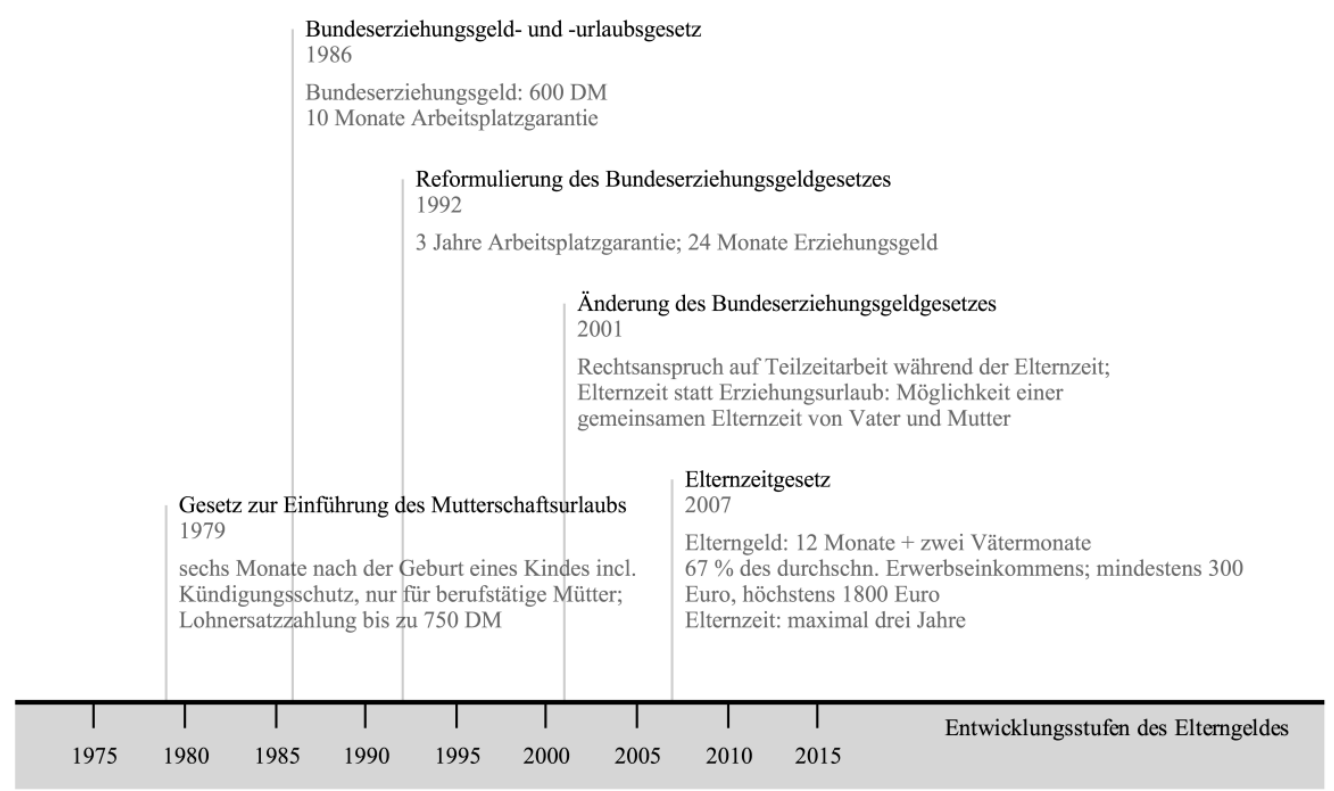

Gerade angesichts dieser Kontinuität in der gesellschaftlichen und politischen Diskussion und in der Entwicklung dieser Modelle stellt sich die Frage, warum der familienpolitischen Maßnahme des einkommensabhängigen Elterngeldes eine solche Bedeutsamkeit zugeschrieben wird. Dies zeigt sich an der großen Aufmerksamkeit, die diese Maßnahme erfahren hat, aber auch an der Fülle von Kontroversen, die sich darum entwickelt haben und die bis heute nicht abgeschlossen sind, wie sich an der Diskussion um das Betreuungsgeld ablesen lässt. Dabei wird auch jenes schon klassische Argument bemüht, der Staat habe nicht das Recht, spezifische Lebensformen besonders zu fördern, sondern müsse nur die Wahlfreiheit zwischen Lebensformen gewährleisten, das bei jeder Veränderung vom Mutterschutz zum Erziehungsurlaub, vom Erziehungsurlaub zur Erziehungszeit und zum einkommensabhängigen Elterngeld immer wieder formuliert wurde.

Im Folgenden wird die These entwickelt, dass die Bedeutsamkeit des einkommensabhängigen Elterngeldes für die Familie vor allem darin zu suchen ist, dass hier erstmalig eine familienpolitische Maßnahme nicht mehr als eine Einzelmaßnahme neben anderen geplant wurde, sondern in das integrative Konzept einer nachhaltigen Familienpolitik eingebettet war. Nachhaltige Familienpolitik nimmt die Leistungen der Familie für die Gesellschaft zum Ausgangspunkt für die Unterstützung der Familie, formuliert auf dieser Grundlage klare Zielvorgaben und ermöglicht und erfordert damit auch die Prüfung der Zielerreichung durch die Evaluation der Maßnahmen. Inhaltlich richtet sich nachhaltige Familienpolitik auf die „Gestaltung der Rahmenbedingungen familiären Lebens mit dem Grundsatz, zukünftigen Generationen die gleichen Chancen zur Gestaltung eigener Lebensvorstellungen und Ziele zu ermöglichen, wie das für die jetzt aktive Generation möglich ist“ (Bertram/Rösler/Ehlert 2005: 6). Als solche ist sie im Wesentlichen eine „Politik zur Zukunftssicherung einer Gesellschaft.“ (ibd.: 9). Sie basiert folglich auf dem konkre- 
ten Anspruch ,junge Erwachsene, Eltern und Kinder so zu unterstützen, das sie das eigene Humankapital entwickeln können, gleichzeitig aber in die eigene individuelle Zukunft und in die Zukunft der Partnerschaft sowie in die Zukunft der eigenen Kinder investieren können“ (ibd.: 9). Um diesem integrativen Anspruch gerecht zu werden muss Familienpolitik auf einem Mix aus Infrastrukturpolitik, Zeitpolitik und finanzieller Transferpolitik aufbauen. Denn nur so lassen sich tatsächlich Rahmenbedingungen schaffen, „die es jungen Erwachsenen ermöglichen, ihre Zukunft gemeinsam und gemeinsam mit Kindern zu planen und zu realisieren" (ibd.: 7).

Historisch war es vermutlich ein Glücksfall, dass mit Renate Schmidt und Ursula von der Leyen zwei Familienministerinnen aufeinander folgten, die aus der unmittelbaren Anschauung ihrer eigenen Lebensentwürfe und ihrer eigenen familiären und beruflichen Situation nicht nur erlebt hatten, dass Familienpolitik aus der Sicht der Betroffenen nur ganzheitlich begriffen werden kann, sondern auch selbst mit den Problemen konfrontiert waren und sind, die die Mehrheit der jungen Frauen heute als qualifizierte Berufstätige zu lösen hat. In diesem Sinne wird Familienpolitik möglicherweise stärker als andere Politikbereiche konkret auch durch die verantwortlichen Akteurinnen und Akteure geprägt. Allerdings wäre das integrative Konzept nachhaltiger Familienpolitik nicht entwickelt und schon gar nicht durchgesetzt worden, wenn es historisch nicht schon viel früher in einzelnen Elementen der Familienpolitik Paradigmenwechsel gegeben hätte, die dann zur Durchsetzung des Konzepts herangezogen werden konnten.

Im Folgenden wird die historische Entwicklung einzelner Aspekte nachhaltiger Familienpolitik bezogen auf die drei Kernelemente Infrastruktur, Zeit und Geld dargestellt, die den Ausgangspunkt für die Formulierung eines ganzheitlichen Konzepts bildeten (Kapitel 2 bis 4). Erst auf diesen Grundlagen wird das einkommensabhängige Elterngeld als ein Baustein einer nachhaltigen Familienpolitik verständlich, welches tatsächlich erweiterte Handlungsspielräume für Mütter, Väter und Kinder herzustellen versucht (Kapitel 5). Allerdings stößt die Umsetzung eines ganzheitlichen Konzepts nachhaltiger Familienpolitik immer noch an Grenzen, insbesondere bezüglich der Ausgestaltung politischer Rahmenbedingungen für die individuelle Organisation des Lebenslaufs von Müttern und Vätern und der eigenständigen Absicherung der ökonomischen Teilhabe von Kindern (Kapitel 6). Das einkommensabhängige Elterngeld ist insofern nur ein Element einer nachhaltigen Familienpolitik.

\section{Infrastruktur als ein Element nachhaltiger Familienpolitik}

Der grundlegende Paradigmenwechsel im Bereich der Infrastrukturpolitik wurde durch die Ablösung des Jugendwohlfahrtsgesetzes (JWG) durch das Kinder- und Jugendhilfegesetz (KJHG) Anfang der 1990er Jahre eingeleitet. Denn das KJHG beinhaltet eine Neuformulierung des Verständnisses von Kindergarten und Krippe weg von der Kompensationsfunktion für mögliche Mängel der Eltern hin zum eigenständigen, ergänzenden Erziehungsaustrag zur Förderung der Teilhabe von Kindern. Das Jugendwohlfahrtsgesetz von 1924 hatte bis in die 1980er Jahre einige Novellierungen erlebt (Sachße 1996); in seinen Grundzügen verpflichtete es die Jugendhilfe zum Einschreiten, wenn spezifische Probleme der Kinder oder Jugendlichen oder der familiären Lebenslagen offenkundig wurden, 
indem der Staat sein Wächteramt ausübte, um mögliche Defizite in der kindlichen Entwicklung zu kompensieren. 1984 schlug der Familien- und Jugendminister Heiner Geißler vor, dieses Gesetz erneut zu novellieren, um es an die geänderte Lebensrealität der Bundesrepublik anzupassen. Doch als Rita Süßmuth 1985 Ministerin wurde, entschied sie kurzfristig, die fast fertige Novelle zu stoppen und ein neues Gesetz auf den Weg zu bringen, nämlich das Kinder- und Jugendhilfegesetz (KJHG bzw. SGB VIII), das 1990 zuerst in den neuen Bundesländern und 1991 in den alten Bundesländern in Kraft trat. Dieses Gesetz betont nicht nur das Recht eines jeden Kindes auf Entwicklung und Erziehung zur eigenverantwortlichen Persönlichkeit, sondern sieht die Eltern an erster Stelle in Verantwortung, um dieses Recht zu verwirklichen, die dabei durch die Kommune, die Zivilgesellschaft und den Staat zu unterstützen sind.

Ebenso wie der parallel zu diesem Gesetz geschriebene Achte Jugendbericht (1990) greift das KJHG damit die klassische Vorstellung von Subsidiarität auf und fragt zunächst nach der kleinsten zuständigen Einheit. Zum anderen folgt es der angelsächsischen Tradition der sozialökologischen Sozialisationsforschung und bietet für den familiären Kontext, in dem Kinder aufwachsen, aktive familienergänzende Leistungen für Kinder und Familien an. Urie Bronfenbrenner (1977) oder James Coleman (1979) betonen immer wieder, dass Familien ihre Sozialisationsleistung zur Entwicklung der kindlichen Persönlichkeit nur dann angemessen erbringen können, wenn die Nachbarschaft, die Gemeinde und der Staat sie darin unterstützen. Aus dieser Sicht ist es verständlich, warum in der Kinder- und Jugendhilfe die freien Träger als Teil der Zivilgesellschaft und die Kommune gegenüber den Ländern und dem Bund den Vorrang haben, wenn es darum geht, die Ressourcen für Eltern und Kinder zu organisieren und die Familie in der Sozialisation ihrer Kinder zu unterstützen. Kindergarten und Krippe füllen unter dieser Perspektive nicht mehr eine Kompensationsfunktion für mögliche Mängel und Fehler der Eltern aus, sondern haben einen eigenständigen familienergänzenden Erziehungsauftrag. Aus diesem Paradigmenwechsel im KJHG leitet sich folgerichtig ein Rechtsanspruch auf diese zusätzlichen Angebote für alle Kinder ab, jedoch keinesfalls eine Pflicht dazu, weil es primär Aufgabe der Eltern ist zu entscheiden, inwieweit diese Angebote die kindliche Entwicklung fördern. Dennoch ist sicherzustellen, dass alle Kinder die Möglichkeit zur Förderung in solchen Einrichtungen auch bekommen können.

Der Rechtsanspruch auf einen Kindergartenplatz für Kinder ab drei Jahren wurde von Angela Merkel als zuständiger Ministerin durchgesetzt (1995); damals kam im Übrigen niemand auf die Idee, ein Betreuungsgeld für die Eltern zu fordern, die ihre Kinder nicht in den Kindergarten geben, denn es ist allein die Entscheidung der Eltern, diese familienunterstützende Leistung in Anspruch zu nehmen oder nicht. Die jüngsten Daten zum Kindergartenbesuch der Drei- bis Sechsjährigen in Deutschland zeigen im Vergleich zum europäischen Ausland, dass dies inzwischen für weit über 90 Prozent aller Kinder selbstverständlich geworden ist (Statistisches Bundesamt 2011; Wolf/Grgic 2009). Dass diese familienergänzenden Maßnahmen damals wesentlich auf Kinder ab drei Jahren konzentriert wurden, hängt auch mit dem seit 1992 bestehenden Anspruch auf den dreijährigen Erziehungsurlaub (Elternzeit) zusammen. Zudem wurde damals in der Fachliteratur heftig die Frage diskutiert, ab wann Kinder vor dem dritten Lebensjahr eine solche Einrichtung besuchen könnten. Längsschnittuntersuchungen zu diesem Thema gab es in Deutschland überhaupt nicht, und die wenigen US-amerikanischen Längsschnitte (NICHD) liefen erst 
wenige Jahre und waren in der Wissenschaft und der Öffentlichkeit noch nicht so bekannt wie heute. Doch ermöglichten der Paradigmenwechsel im KJHG zusammen mit der wissenschaftlichen Erkenntnis (u.a. Shonkoff/Phillips 2000), dass sich die Entwicklungsperspektiven von Kindern vor allem im Umgang mit anderen Kindern auch schon früher fördern lassen, eine sehr offene und von allen Parteien letztlich akzeptierte Weiterentwicklung des Anspruches von Kindern auf familienunterstützende Leistungen auch vor dem dritten Lebensjahr.

Das Tagesbetreuungsausbaugesetz, das Renate Schmidt als Familienministerin vorangebracht hat, baut auf den Diskussionen und Entwicklungen seit 1990/95 auf und trat 2005 in Kraft. Es erhöhte die Plätze für die unter dreijährigen Kinder nicht nur um weitere 230.000 Plätze bis 2010, sondern nahm auch deutlich Bezug auf den Erziehungsanspruch im KJHG und definiert klare Qualitätsansprüche an Kindertagesstätten, Kinderkrippen und Tagesmütter. Ursula von der Leyen führte diesen Gedanken fort: 2007 beschloss das Bundeskabinett, durch den Ausbau der Plätze für die unter dreijährigen Kinder auf 750.000 Plätze bis 2013 auch den Rechtsanspruch für Kinder unter drei Jahren auf solche familienunterstützenden Leistungen sicherzustellen. Die Umsetzung dieser Maßnahmen ist Aufgabe der Kommunen zusammen mit den freien Trägern vor Ort, ohne dass sie für diese zusätzliche Aufgabe ausreichend Finanzmittel von Bund oder Ländern bekamen. Zwar hat der Bund zusätzliche Mittel zur Verfügung gestellt, diese decken aber den Finanzbedarf nicht.

Abbildung 2: Die Entwicklung von Infrastruktur in Deutschland

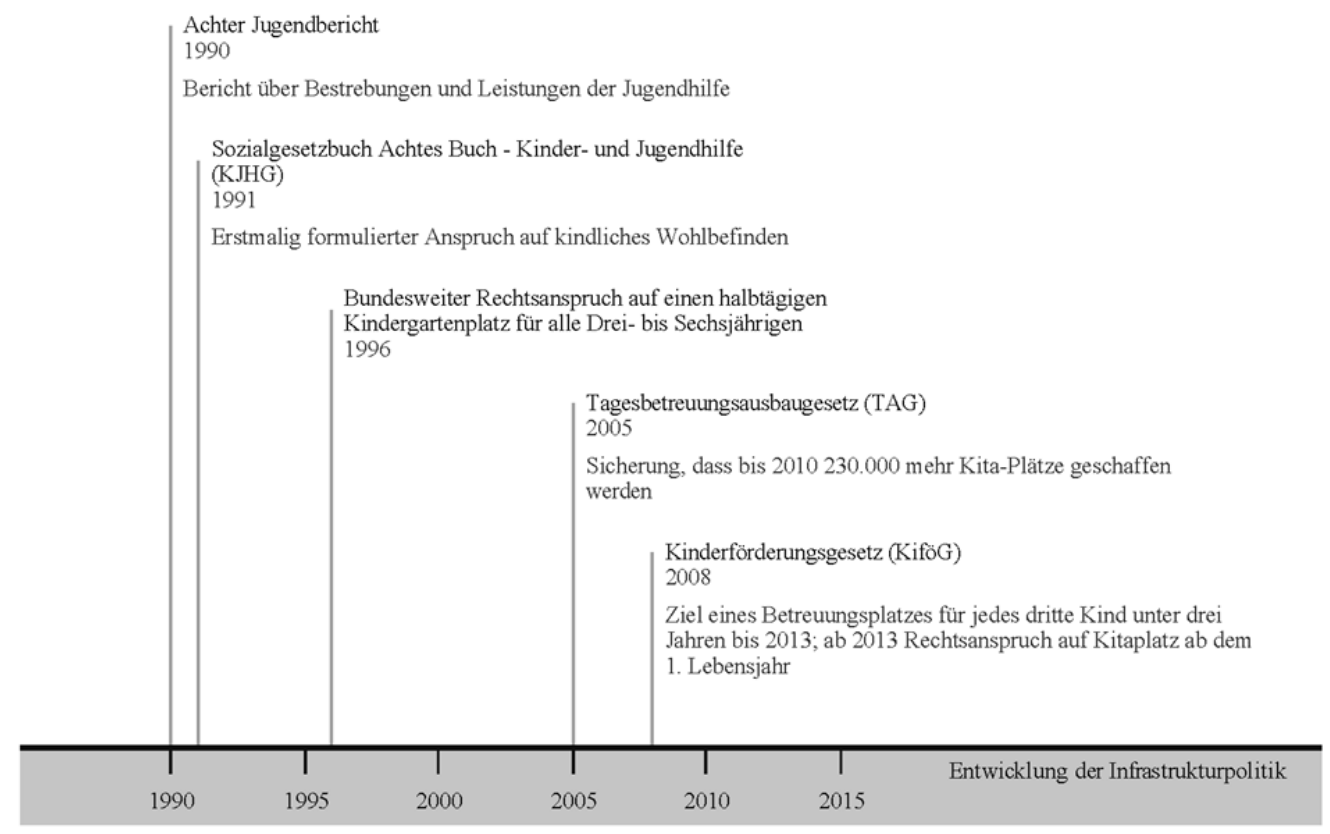




\section{Alltagszeit und Lebenszeit als zentrale Elemente nachhaltiger Familienpolitik}

Der systematische Ausbau des Betreuungsangebots zunächst für Kinder im Kindergartenalter und später für Kinder ab dem zweiten Lebensjahr baut zunächst auf der pädagogischen Grundlinie des KJHG auf, dass jedes Kind ein Recht auf Förderung seiner Entwicklung hat und die Eltern bei der Sozialisation ihrer Kinder von Nachbarschaft, Gemeinde und Staat zu unterstützen sind. Er folgt aber auch der Einsicht, dass bei der gewandelten ökonomischen Struktur der modernen Gesellschaft nicht mehr auf die berufliche Integration der inzwischen hoch qualifizierten jungen Frauen und Mütter verzichtet werden kann. Dies hat für die Familienpolitik wiederum zur Konsequenz, dass eine nachhaltige Strategie die Schaffung gesellschaftlicher Rahmenbedingungen erforderlich macht, die jungen Müttern und Vätern die zeitliche Vereinbarkeit von Erwerbstätigkeit und familiärer Fürsorge auch tatsächlich ermöglichen. Dies betrifft sowohl die Organisation der Alltagszeit von Familien als auch die Integration von Fürsorgezeiten in den Lebensverlauf. Für die Umsetzung einer solchen familienpolitischen Strategie sind Fragen der Kinderbetreuung, aber auch die Schaffung eines familienfreundlichen Arbeitsrechts, die betriebliche Anpassung an die Bedürfnisse von Müttern und Vätern sowie kommunale, zivilgesellschaftliche Akteursstrategien zentral.

Solange die klassische Arbeitsteilung der Industriegesellschaft in der Familie bestand stellte sich diese konkrete Frage nach der Organisation der Alltagszeit nicht (Bertram 1997): Die private Lebensführung wurde von der Hausfrau und Mutter erledigt und der erwerbstätige Mann konnte sich den Bedingungen der betrieblichen Produktion anpassen. Anders als andere europäische Länder ist Deutschland mit einem recht hohen Anteil an Industriearbeitsplätzen und industrienahen Dienstleistungen verhältnismäßig industriegesellschaftlich geblieben. In diesen Bereichen richten sich noch heute die Arbeit und die Arbeitszeiten nach der Prozessstruktur der Produktion, die die privaten Verhältnisse der Beschäftigten a priori nicht berücksichtigen. Daher ist es nicht verwunderlich, dass die Länder mit einem höheren Anteil an Beschäftigten im öffentlichen Dienst, wie Frankreich oder Schweden, oder mit einem deutlicheren Abbau von Industriearbeitsplätzen, wie die USA oder Großbritannien, die Vereinbarkeit von Fürsorge für Kinder und beruflichen Aktivitäten vorantreiben konnten. Dennoch hat Deutschland bei der Beschäftigungsquote von jungen Frauen mit Kindern mit den anderen europäischen Ländern, etwa Frankreich, inzwischen fast gleichgezogen (Bertram 2012). Das hängt möglicherweise mit den gesetzlichen Rahmenbedingungen für den Anspruch auf Teilzeitbeschäftigung seit 2001 zusammen, die die Ministerin Christine Bergmann gesetzlich regelte. Diese eröffneten Eltern die Möglichkeit ihre Bedürfnisse zwischen der Fürsorge für Kinder und den beruflichen Anforderungen auf Basis eines eigenen Rechtsanspruch mit dem Arbeitgeber zu verhandeln.

Aber die gesetzlichen Rahmenbedingungen stellen immer nur eine Voraussetzung solcher Entwicklungen dar. Daher gründete Renate Schmidt als nachfolgende Ministerin eine „Allianz für Familien“, damit die wichtigsten Akteure des Arbeitsmarkts zusammen mit den Wohlfahrtsverbänden und der Politik auch betriebliche Lösungen entwickelten, um unter den gegebenen gesetzlichen Bedingungen, den betrieblichen Erfordernissen und den Möglichkeiten zur Fürsorge durch individuelle Regelungen einen Interessenausgleich zwischen Arbeitgebern und Familien zu organisieren. Dieses zivilgesellschaftliche Enga- 
gement bei der Neukonzeption von betrieblicher Zeitorganisation unter Berücksichtigung auch der Bedürfnisse von Familien sollte in seiner Wirkung schon deswegen nicht unterschätzt werden, weil die Möglichkeiten des Gesetzgebers, individuelle Lösungen für einzelne Betriebe vorzugeben, begrenzt sind. Wenn aber die Spitzen der Industrie- und Handelskammern, der Gewerkschaften, des Bundesverbandes der Industrie, um nur einige zu nennen, sich für solche Ziele in die Pflicht nehmen lassen, hat ein solches zivilgesellschaftliches Engagement auch für die einzelnen Betriebe erhebliche Wirkung. Denn dadurch ändern sich auf Dauer die Werte und Vorstellungen, wie Familie und Beruf in Einklang zu bringen sind. Neben dieser „Allianz für Familien“ wurden auch „Bündnisse für Familien“ (BMFSFJ 2010) gegründet, auf der Basis der im KJHG vorgezeichneten Idee, auf kommunaler Ebene Menschen aus ganz unterschiedlichen Bereichen vor Ort zu motivieren, sich gemeinsam für eine bessere Betreuung und Unterstützung von Familien in verschiedenen institutionellen Kontexten einzusetzen.

Innerhalb weniger Jahre entstanden mehrere hundert solcher lokaler „Bündnisse für Familien", sie waren insofern recht erfolgreich. Damit wurde nicht nur auf der obersten Ebene von Politik und Verbänden die Frage der Vereinbarkeit von Familie und Beruf thematisiert, sondern auch ganz konkret in der Nachbarschaft und der Gemeinde, wie dies im Konzept der Subsidiarität des KJHG angelegt ist. Allerdings hat Kurt Biedenkopf darauf hingewiesen, dass diese Form von Subsidiarität strukturell vom klassischen Modell der Subsidiarität zu differenzieren ist. Das klassische Modell ging davon aus, dass die kleinste Einheit ihre Angelegenheiten allein und selbstständig erledigen kann und die nächst höhere Einheit, ob Kommune, Bundesland oder Bund, nur dann eingreift, wenn sie es nicht selbst kann. Diese neue Form der Subsidiarität stellt hingegen konzeptionell ein Wechselspiel zwischen zivilgesellschaftlichem Engagement und kommunalen, länderspezifischen oder bundesspezifischen Angeboten dar (Biedenkopf/Bertram/Niejahr 2009).

Dabei ersetzen die „kleinen Lebenskreise“ nicht die staatlichen Angebote und übernehmen auch nicht von der Kommune oder dem Land organisierte Angebote, sondern entwickeln weitere Möglichkeiten der Kooperation zwischen verschiedenen Lebensbereichen im Interesse der vielfältigen und unterschiedlichen Bedürfnisse von Familien und Kindern im kommunalen Kontext (ibd.). Das mag in einem Fall heißen, einen Industriebetrieb zu überzeugen, bestehende kommunale Infrastrukturen für Kinder mit zu unterstützen, damit auch Betriebsangehörige diese Infrastrukturen nutzen können. Oder man verständigt sich gemeinsam darauf, die Orte für Kinder so zu organisieren, dass alle kindlichen Aktivitäten möglichst an diesem einem Ort stattfinden können, was die Kooperation der verschiedenen Akteure voraussetzt. Ohne dieses zivilgesellschaftliche Engagement sowohl auf der Verbandsebene wie auch vor Ort in den Bündnissen für Familien wäre der rasche Ausbau der Betreuung für die unter Dreijährigen kaum zu bewältigen gewesen, weil die Bündnisse für Familien jetzt diese Themen auch auf die Agenda der Kommunalpolitik setzen und dafür sorgen, dass sie Thema der Kommunalpolitik bleiben.

Diese Strategie zur Förderung des zivilgesellschaftlichen Engagements ist bei der Infrastruktur der Bundesrepublik als einem föderalen Staat besonders zu berücksichtigen. Denn in Frankreich als einem Zentralstaat versprach beispielsweise Präsident Mitterrand zu Beginn seiner Amtszeit (1981) 300.000 neue Krippenplätze und hatte am Ende seiner Amtszeit gerade 60.000 umgesetzt (Michel/Mahon 2002). Die sich jetzt abzeichnende Entwicklung der Infrastruktur für Kinder in Deutschland mit Betreuungsplätzen für ein 
Drittel der unter dreijährigen Kinder, und dies innerhalb von sechs bis sieben Jahren, ist vermutlich eine der ganz großen sozialpolitischen Leistungen in Deutschland. Das wäre nicht möglich gewesen, wenn beim Ausbau der Infrastruktur für Kinder diese Möglichkeiten der Einbindung der zivilgesellschaftlichen Akteure auf den verschiedenen Ebenen der Gesellschaft nicht konzeptionell mitgedacht und mit großer politischer Energie und großem politischen Einsatz unter Einschluss der großen Stiftungen in Deutschland vorangebracht worden wären. Selbst wenn 2013 die versprochenen 750.000 Plätze nicht vollständig zur Verfügung stehen, war die Entwicklung dieser Infrastruktur für die Vereinbarkeit von Familie und Beruf auf jeden Fall ein zentrales Element, weil nur so die Abstimmung von Zeit für Fürsorge und Zeit für Beruf gesellschaftlich gelöst werden konnte. Ohne diesen Wandel wäre die Organisation der Alltagszeit und der deutlich zunehmenden außerhäuslichen Erwerbstätigkeit von Müttern mit Kindern kaum zu bewältigen gewesen.

Abbildung 3: Zeitpolitik und Flexibilisierung von Arbeitszeit und Familienzeit

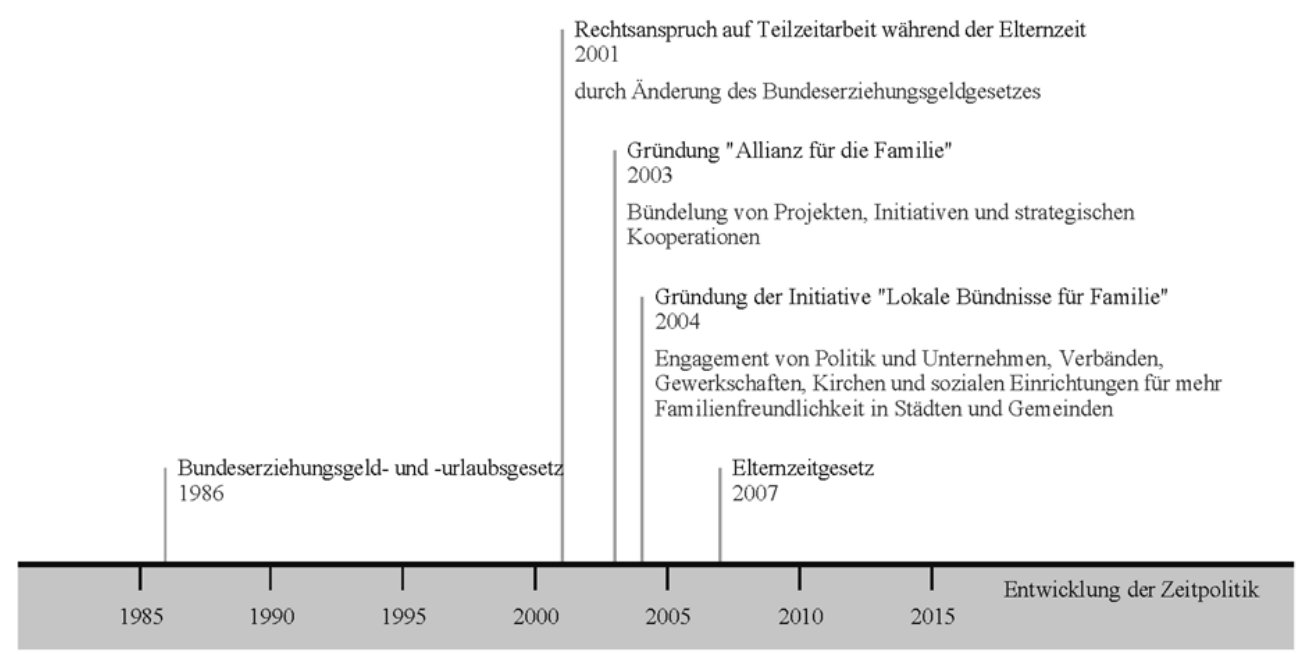

Diese Strategie, die Themen Zeit für Kinder und Arbeitszeit für Eltern durch klare gesetzliche Vorschriften und durch die Einbindung der zentralen Akteure am Arbeitsmarkt und zugleich der lokalen Akteure zu verknüpfen, hat sicherlich wesentlich dazu beigetragen, dass dieses Thema inzwischen selbst in überwiegend männerdominierten Industriebereichen selbstverständlich geworden ist. Doch ist nicht zu verkennen, dass ein anderes großes Thema, nämlich die Organisation von Lebenszeit, trotz der Empfehlungen der EU (Kommission der Europäischen Gemeinschaften 2005), bis heute in Deutschland noch nicht zu einem großen Thema geworden ist. Das hängt damit zusammen, wie später noch gezeigt wird, dass es bisher nicht gelungen ist, die Politik für Familien und Kinder als eine lebensverlaufsorientierte Politik zu konzipieren, wie es der Siebte Familienbericht (2006) vorgeschlagen hat. Auch beim Ausbau der Kinderbetreuung und der Frage der zeitlichen Organisation von Arbeit und Familie dominiert ein problembezogener Ansatz, der die aktuelle Alltagszeit und die aktuellen Alltagsprobleme in den Mittelpunkt der Be- 
trachtung stellt und nicht fragt, ob nicht andere Modelle in einem Karriereverlauf einen Teil dieser Zeitprobleme lösen könnten.

\section{Familienlastenausgleich oder Familienleistungsausgleich: Geld und nachhaltige Familienpolitik}

Im Bereich der finanziellen Transferleistungen für Familien deutet sich ein Paradigmenwechsel von einem kompensatorischen zu einem leistungsorientierten Ansatz bisher noch nicht an. Das kompensatorische Verständnis finanzieller Transferleistungen, welches bei der Unterstützung von Familien dem Prinzip des Nachteilsausgleichs folgt, bildet nach wie vor den Maßstab der Leistungen für Familien. Ein teilhabeorientiertes Verständnis finanzieller Transferleistungen würde demgegenüber positiv die Leistungen, die Familien für die Gesellschaft erbringen, zum Maßstab der Förderung nehmen. Dies impliziert auch eine Interpretation und Sicherung der ökonomischen Kosten für Kinder als völlig eigenständig und unabhängig von den Eltern.

Schon der Schreiberplan von 1955 (Schreiber 2004 [1955]) beinhaltete solch eine Vorstellung der unabhängigen ökonomischen Sicherung der nachwachsenden Generation. Die Einführung der dynamischen Alterssicherung 1957 auf der Basis des Schreiberplans sollte sicherstellen, dass auch die Rentner, etwa die Kriegerwitwen, angemessen an dem sich abzeichnenden wirtschaftlichen Aufschwung der Bundesrepublik partizipieren (Habisch 1999). Schreiber (2004 [1955]) hat in seinem Plan aber nicht nur eine dynamische Alterssicherung sondern gleichzeitig auch eine dynamische „Vorrente“ für die nachwachsende Generation gefordert, weil ein Umlagesystem der Rente notwendigerweise auf einem DreiGenerationenvertrag basiert: Die älteste Generation wird durch die mittlere Generation gesichert, und die durch die Arbeit dieser Generation gesicherten Ansprüche werden später wiederum durch die nachwachsende Generation abgesichert; nach Schreibers Idee sollte diese Vorrente später im aktiven Berufsleben zurückgezahlt werden. Andere Länder, wie Frankreich oder Schweden, kennen solche Finanzierungen der nachwachsenden Generation, jedoch wurde diese Idee in der Bundesrepublik 1957 nicht weiterverfolgt, weil sich die beiden großen Parteien CDU und SPD bei den Rentenversprechen wechselseitig überboten. Im Modell der Vorrrente ist der Gedanke angelegt, dass Kinder und Familien zu unterstützen sind, weil die Gesellschaft von ihnen später erwartet, die Beiträge für die Rente zu erwirtschaften, um der dann inaktiven Generation ein würdiges Alter zu ermöglichen.

Diese Vorstellung, Geld aus der Sozialversicherung zur Verfügung zu stellen, um zukünftige Rentenleistungen zu sichern, findet sich später in der neuen Haushaltsökonomie von Gary S. Becker wieder; er verweist darauf, dass Kinder in historischer Perspektive immer als Alterssicherung der eigenen Eltern und Großeltern galten (Becker 1976). Obwohl Becker dafür den Nobelpreis bekam, hat sich dieser Leistungsgedanke und diese Drei-Generationen-Beziehung in der deutschen Politik nicht durchsetzen können. Zwar hat der Fünfte Familienbericht (1995) erreicht, was inzwischen auch politisch anerkannt ist, dass in den Familien die Basis für das Humanvermögen der Gesellschaft gelegt wird, weil diese Drei-Generationen-Beziehung ohne Kinder und ohne die Erziehungsleistung der Eltern nicht zu realisieren ist. 
Doch trotz der klaren wissenschaftlichen Begründung und Nobelpreis blieb die deutsche Politik bei der Unterstützung von Familien dem Prinzip des Nachteilsausgleichs treu, der schon beim ersten Kindergeld und beim Ehegattensplitting das Grundprinzip war. Heute wird vom Familienleistungsausgleich gesprochen, doch ist das Ehegattensplitting seiner Grundkonstruktion nach der Versuch, das Paar als eine wirtschaftliche Einheit wie eine Gesellschaft bürgerlichen Rechts (GbR) zu interpretieren und den Nachteil durch die Addition beider Einkommen in der Steuerprogression dadurch auszugleichen, dass das Einkommen halbiert wird. Das Kindergeld ist unabhängig von seiner Höhe ähnlich konstruiert, weil es den Familien gezahlt wird, die wegen ihres geringen Einkommens nicht in den Vorteil von Steuerfreibeträgen kommen, die bei einer progressiven Steuer erforderlich sind, um die Leistungsfähigkeit des Einzelnen in der Steuer gerecht und richtig zu erfassen. Wenn der Steuerpflichtige Unterhaltsverpflichtungen gegenüber Kindern hat, wird seine steuerliche Leistungsfähigkeit durch diese Verpflichtungen entsprechend vermindert. Die Durchsicht der verschiedenen familienpolitischen Leistungen (BMFSFJ 2009), die 2010 beispielsweise immerhin 125,5 Milliarden Euro familienbezogener zuzüglich 74,9 Milliarden Euro ehebezogener Leistungen betrugen (BMFSFJ 2013), führt zu dem Ergebnis, dass fast alle Regelungen, ob die Anerkennung von Erziehungszeiten in der Rente oder die beitragsfreie Mitversicherung von Ehefrauen, von dem Grundgedanken geprägt sind, die Nachteile, die durch die Lebensform von Ehe und Familie für den Einzelnen entstehen können, finanziell auszugleichen.

Demgegenüber wählen Schreiber und Becker in ihrer neuen Haushaltsökonomie ähnlich wie der Fünfte Familienbericht einen anderen Ansatz, nämlich die Unterstützung von Familien nicht als Nachteilsausgleich zu konzipieren, sondern positiv die Leistungen, die die Familien für die Gesellschaft erbringen, zum Maßstab der Familienförderung zu machen. Dabei geht es nicht nur um die Entwicklung des Humanvermögens, sondern auch um die Pflegeleistungen für die ältere Generation, die in Deutschland wesentlich familiär organisiert sind. Die Fürsorge für andere als zentrales Element des privaten Lebens kann von staatlichen Institutionen nur in Ansätzen geleistet werden, weil das Grundprinzip dieser Fürsorge die private, persönliche und intime Beziehung und das unmittelbare Vertrauen zwischen den Personen ist. Die internationale Literatur betont diesen Zusammenhang sowohl hinsichtlich der Kinder (Waldfogel 2006; Waldfogel/Craigie/Brooks-Gunn 2010; Belsky 2012; Ahnert/Lamb 2011), als auch hinsichtlich der älteren Generation, die privatpersonale Fürsorge und Unterstützung als ein wesentliches Element der eigenen Lebenszufriedenheit interpretiert, wie viele empirische Untersuchungen zeigen (Bertram 1991; Kohli/Hank/Künemund 2009; Börsch-Supan 2009).

Bei den finanziellen Leistungen für die Familie ist es demgegenüber bis heute nicht gelungen, ein solches leistungsbezogenes Konzept durchzusetzen. Vermutlich wird man hier auf das Bundesverfassungsgericht warten müssen, das 2010 in einem Urteil (BVerfG 2010) bereits angemahnt hat, dass die Berechnung der ökonomischen Unterstützungsleistungen für Kinder nicht vom Anspruch für Erwachsene abgeleitet werden kann, sondern eigenständig und unabhängig zu berechnen ist. Das ist ein erster Schritt, die den Eltern entstehenden ökonomischen Kosten von Kindern als völlig eigenständig und unabhängig von den Erwachsenen zu interpretieren. Politisch ist das vermutlich nur in Form einer „Grundsicherung“ durchzusetzen mit der Konsequenz, dass kein Nachteilsausgleich finanziert wird, der der jeweiligen politischen Einschätzung des Nachteils oder auch der 
Kassenlage des Bundes unterliegt, sondern eine unabhängige Existenzsicherung, wie sie Schreiber schon vor 60 Jahren empfahl.

Abbildung 4: Die Entwicklung finanzieller Unterstützungen für die Familie

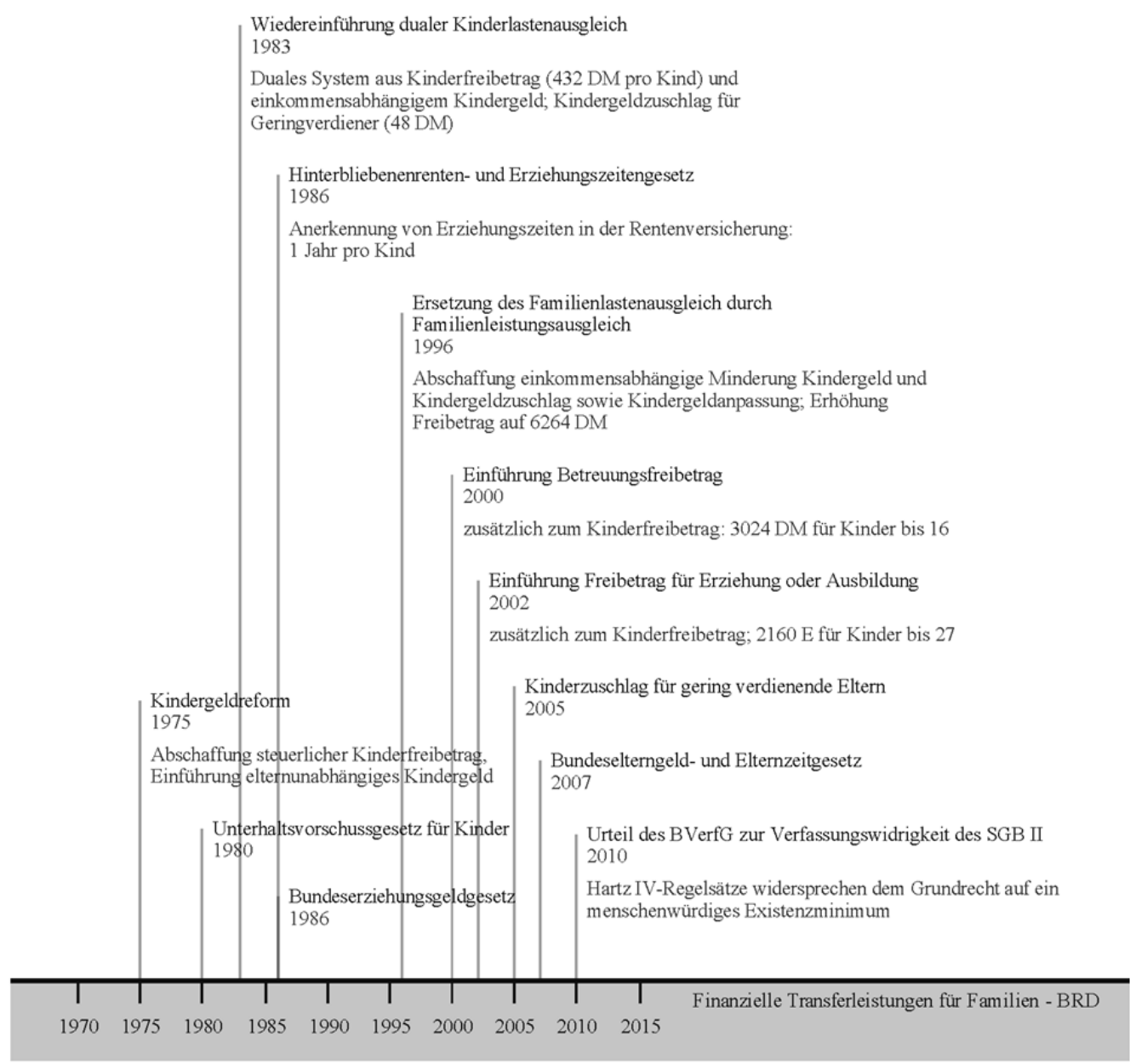

\section{Das einkommensabhängige Elterngeld als ein Baustein nachhaltiger Familienpolitik}

Das Elterngeld wird im Siebten Familienbericht (BMFSFJ 2006) in verschiedenen Varianten ausführlich dargestellt und in seiner heute gültigen Form im Einzelnen begründet. Es lässt sich in seiner Ausgestaltung aus bestimmten politischen Entwicklungen in der Sozial- und Familienpolitik ableiten. Die Entscheidung, das Elterngeld einkommensab- 
hängig zu zahlen und an den aktuellen Lohn zu binden, folgt zunächst einmal dem Prinzip der Sozialgesetzgebung, dass die erreichte Lohnhöhe der Maßstab für Lohnersatzleistungen und Rente ist. Zentraler Grundgedanke des Elterngeldes ist die Vorstellung, dass die Fürsorge für Kinder im ersten Lebensjahr durch die Eltern eine notwendige Voraussetzung für die Entwicklung und Erziehung von Kindern zu gemeinschaftsfähigen Persönlichkeiten und für ihre Teilhabe an der gesellschaftlichen Entwicklung darstellt. Diese Fürsorge ist eine Leistung, die die Eltern für die Kinder erbringen, aber damit zugleich eine zentrale gesellschaftliche Aufgabe ausüben. Daraus ist abzuleiten, dass diese Leistung den gleichen Stellenwert in der modernen Gesellschaft hat wie die Teilhabe am Arbeitsmarkt, die zunächst der persönlichen ökonomischen Existenzsicherung dient, aber darüber hinaus immer auch eine notwendige Voraussetzung für die ökonomische Entwicklung der gesamten Gesellschaft ist. Damit ist mit dem einkommensabhängigen Elterngeld auch der Anspruch formuliert, diese Fürsorgeleistungen genauso zu bewerten wie die Arbeitsleistungen am Arbeitsmarkt.

In dieser starken Begründung des einkommensabhängigen Elterngeldes für Fürsorge finden sich einerseits die Grundlinien des KJHG wieder, die die Entwicklung der kindlichen Persönlichkeit als zentrale Aufgabe der Eltern betonen, die dabei von der Gesellschaft zu unterstützen sind. Zum anderen ist damit auch die Position zu begründen, dass es bei diesen Leistungen für Familien nicht um einen Nachteilsausgleich gehen kann, sondern um eine positive Begründung von Leistungen der Familie für die Gesellschaft. Daraus ist auch abzuleiten, dass die Gesellschaft diese Leistungen nur solange ökonomisch unterstützen muss wie sie unbedingt erforderlich sind, denn die gesellschaftlichen Ressourcen sind knapp und müssen entsprechend wirkungsvoll eingesetzt werden.

Der Zweite Familienbericht (1975) hatte auf dem Stand des damaligen Wissens noch die Auffassung vertreten, dass die elterliche, insbesondere die mütterliche Fürsorge in den ersten drei Lebensjahren ausschließlich erforderlich sei. Inzwischen hat die wissenschaftliche Forschung einschließlich der internationalen Vergleiche deutlich gemacht, dass das erste Lebensjahr für den Aufbau von Bindungen und Beziehungen zwischen Kind und Eltern und damit für die Entwicklung des Urvertrauens des Kindes zur Welt von zentraler Bedeutung ist. Die Erweiterung der kindlichen Beziehungen zu Erwachsenen außerhalb der Familie ab dem ersten Lebensjahr dürfte aller Wahrscheinlichkeit nach keine größere Probleme bereiten, wenn die Qualität der Betreuungseinrichtungen hinsichtlich Personalschlüssel und Stabilität der Betreuungspersonen gesichert sind (Shonkoff/Phillips 2000). Die klare Zielorientierung, das einkommensabhängige Elterngeld nur in der Zeit zu zahlen, in der nach gegenwärtigem wissenschaftlichen Wissen die Fürsorge durch die Eltern selbst von besonderer Bedeutung ist, zeigt deutlich, dass hier die Leistungen der Familie für die Gesellschaft unterstützt werden, was als Prinzip im Fünften Familienbericht (1995) begründet wird.

Bei den Diskussionen um die Ausgestaltung des Elterngeldes dienten vor allem die nordeuropäischen Länder Schweden und Finnland als Beispiele. Der internationale Vergleich hat den unbestreitbaren Vorteil, die Wirkungen dieser Regelungen in den jeweiligen Ländern unter verschiedenen Aspekten untersuchen und mit den bisherigen deutschen Entwicklungen vergleichen zu können. Aus Abb. 5 aus dem Siebten Familienbericht (BMFSFJ 2006: 287) geht hervor, dass eine nachhaltige Familienpolitik mit klaren Zielvorgaben auch die Möglichkeit eröffnet, diese Wirkungen im Einzelnen zu prüfen. Bei einem im Januar 2005 geborenen Kind führte die damalige Regelung von Mutterschutz 
und Elterngeld dazu, dass die ökonomische Situation der Mutter sich zunächst kurzfristig verbesserte, sich danach aber drastisch verschärfte. Dieses Modell mit der zweijährigen Bezugszeit und einem Erziehungsurlaub von insgesamt drei Jahren zeigt den typischen Achterbahneffekt, den Jan Hoem schon 1989 als ein zentrales Problem für junge Familien kritisiert hatte. Beim schwedischen Modell, das 80 Prozent des letzten erzielten Einkommens für 14 Monate ermöglicht, wird ein solcher Achterbahneffekt für die Familien dann vermieden, wenn danach die Erwerbstätigkeit wieder aufgenommen wird.

Abbildung 5: Monatlicher Leistungsbezug von unverheirateten Frauen der Gehaltsgruppe BAT IIa bis 36 Monate nach der Geburt des ersten Kindes nach ausgewählten europäischen Modellen. Fiktiver Geburtstermin:

1. Januar 2005

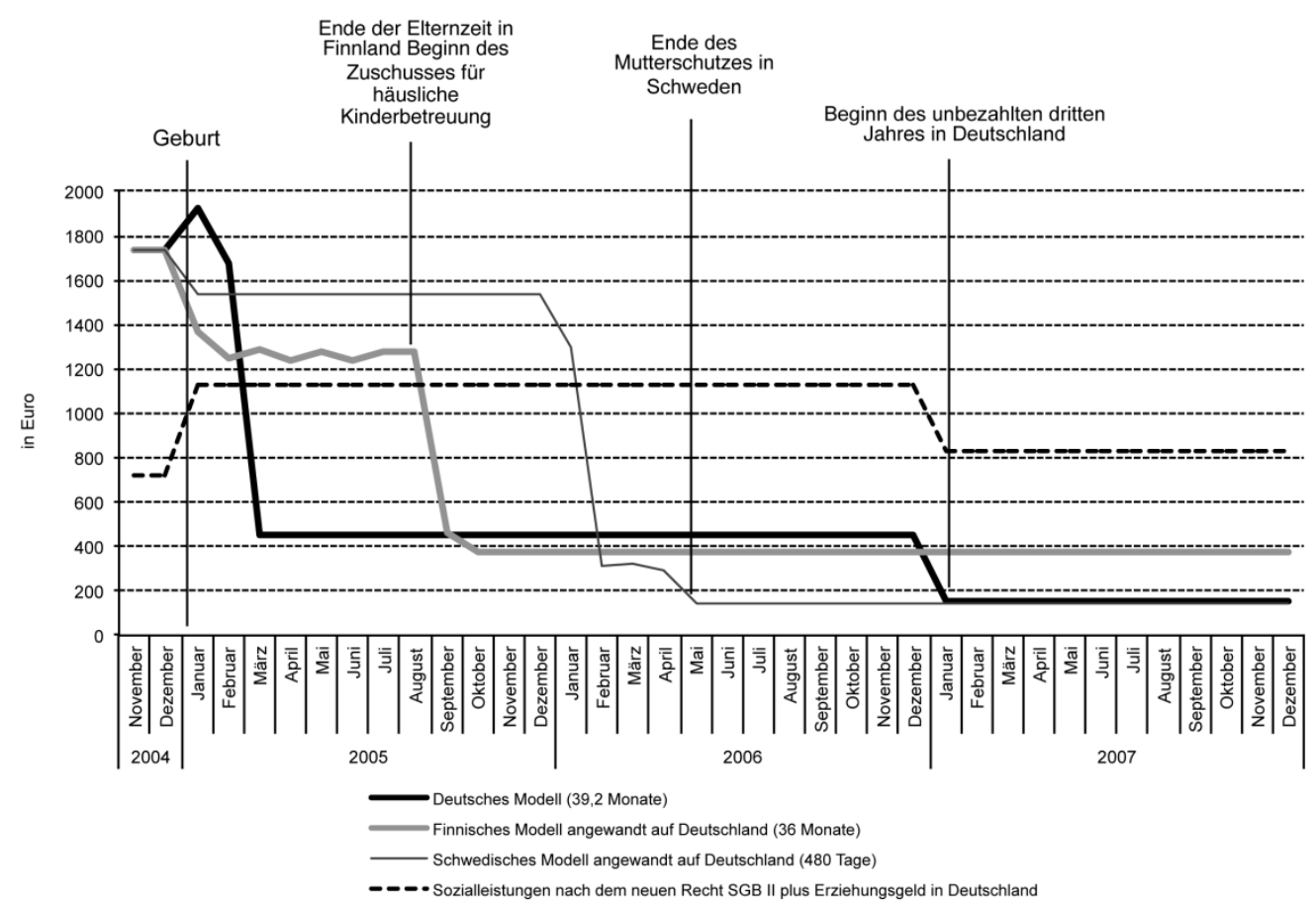

Quelle: eigene Berechnung und Darstellung; zuerst veröffentlich in BMFSFJ 2006: 287.

Als „Achterbahneffekt“ (Hoem/Hoem 1996) wird der Prozess bezeichnet, dass nach der Geburt eines Kindes in der Zeit, in der das Kind unmittelbare Fürsorge braucht, erhebliche Einkommensverluste auftreten bei gleichzeitigem Mehrbedarf wegen des Kindes, die nach Wiederaufnahme des Berufes wieder ausgeglichen werden, um bei einem zweiten oder dritten Kind erneut einzutreten. Hoem war davon überzeugt, dass diese ökonomisch unsichere Situation auch einen negativen Effekt auf die Geburtenrate hat. In Finnland werden 68 Prozent des letzten Einkommens für neun Monate gezahlt, sodann den Müttern, die ihre Kinder nicht in die Krippe geben, für insgesamt drei Jahre ein Betreuungs- 
geld von 400 Euro. Interessanterweise war die günstigste Variante das deutsche Modell aus Sozialleistungen und dem damaligen Erziehungsgeld.

Das heutige einkommensabhängige Elterngeld und die Elternzeit in Deutschland stellen eine Kombination aus dem finnischen und schwedischen Modell dar: In der Zeitdauer ist es am schwedischen Modell orientiert und in der Höhe am finnischen Modell. Im ersten Schritt wurde dieses Modell 2007 in Kraft gesetzt. Im Koalitionsvertrag der jetzigen Bundesregierung wurde dann doch festgehalten, nach erfolgtem Ausbau der Kinderbetreuung auch ein Betreuungsgeld zu zahlen. Die gegenwärtige erbitterte Debatte um das Betreuungsgeld hat vermutlich hier ihren Ursprung, denn die Begründung des Betreuungsgeldes ist auch dem finnischen Modell entnommen, es nämlich nur dann zu zahlen, wenn keine Betreuungseinrichtungen in Anspruch genommen wird.

Nun passt das Betreuungsgeld konzeptionell nicht systematisch in das Konzept einer nachhaltigen Familienpolitik, weil eine solche Familienpolitik auf die Zielorientierung und Integration der verschiedenen familienpolitischen Leistungen hin angelegt ist. Das bedeutet, dass Staat und Gesellschaft bei der Fürsorge für Kinder nur dann ökonomische Ressourcen zur Verfügung stellen, wenn es bei der jeweiligen Maßnahme eine klare Zieldefinition für die Entwicklung der Kinder gibt. Denn eine nachhaltige familienpolitische Strategie legt den Ausbau der angebotenen Infrastruktur quantitativ und qualitativ so fest, dass die Zeit für Fürsorge, die aus wissenschaftlicher und politischer Sicht primär nur von den Eltern selbst zu leisten ist, ökonomisch so gesichert ist, dass der Achterbahneffekt vermieden wird. Das ist dann gewährleistet, wenn nach dieser Zeit für Fürsorge die Berufstätigkeit wieder aufgenommen werden kann, so dass hier eine entsprechende qualitativ und quantitativ gute Infrastruktur zur Verfügung stehen muss.

Gleichzeitig muss die Arbeitszeit so organisiert sein, dass die Lebens- und Fürsorgebedürfnisse von Kindern entsprechend ihrer individuellen Entwicklungsbedingungen auch gesichert sind. Das gelingt am ehesten dann, wenn nicht nur die Mutter diese Fürsorgeleistungen erbringt, sondern auch der Vater in diesen Prozess eingebunden ist. Vater und Mutter können sich die Kombination von Fürsorgezeit, Arbeitszeit und außerfamiliärer Betreuungszeit dann gemeinsam so einteilen, dass alle Beteiligten, nämlich die Mütter, Väter und Kinder, auch subjektiv das Gefühl haben, die Aufgaben in den unterschiedlichen Lebensbereichen angemessen bewältigen zu können. Da es nicht zur klassischen Rolle des Vaters gehört, die Fürsorge für Kinder nicht nur ökonomisch, sondern ganz konkret im Alltag zu leisten, ist es für diesen Rollenwandel erforderlich, die Elternzeit so auszugestalten, dass auch väterliche Fürsorgezeit in der Familie geleistet und als ein Rechtsanspruch gegenüber dem Betrieb formuliert werden kann. In dieses integrative Konzept, das die Zeit der Fürsorge für Kinder ökonomisch stützt, ein hinreichendes und qualitativ gutes Betreuungsangebot zur Verfügung stellt und zudem sicherstellt, dass sich auch die Väter an der Fürsorgezeit beteiligen, passt das Betreuungsgeld nicht.

An der Kombination aus der ökonomischen Unterstützung der Familie bei der Fürsorge für Kinder, dem Ausbau der Infrastruktur, um die Eltern in diesem Prozess zu unterstützen, der Einbindung der Väter und der Abstimmung der Zeiten zwischen Beruf, Familie und Partnerschaft ist das Konzept der nachhaltigen Familienpolitik relativ gut zu rekonstruieren. Die klare Zieldefinition führt dazu, dass bisher relativ unverbundene Maßnahmen wie Kinderbetreuung, ökonomische Unterstützung sowie Arbeitszeit und Zeitorganisation als aufeinander bezogene Elemente gelten, die insgesamt aufeinander abzustimmen sind. 


\section{Nachhaltige Familienpolitik als Lebensverlaufspolitik}

Die Zahlung eines Elterngeldes, der Ausbau der Kinderbetreuung für die unter Dreijährigen und die Regelungen bei den Vätermonaten sind als Einzelmaßnahmen nicht neu. Neu ist der klare Zusammenhang bei dem Anspruch, diese Leistungen in Verbindung mit anderen Familienleistungen so zu organisieren, dass sich unterschiedliche politische Ziele in diesem Kontext realisieren lassen. Das wird an den Vätermonaten deutlich, hinter denen auch der Anspruch steht, dass die Väter eine neue Interpretation ihrer eigenen Rolle innerhalb der Familie finden. Auch die Selbstverpflichtung des Gesetzgebers, in kurzer Zeit eine hinreichende Anzahl von Betreuungsplätzen mit den notwendigen Qualitätsstandards zu schaffen, macht deutlich, dass die Verbesserung der Leistungen von den Familien für den Staat und die Gesellschaft nicht durch einzelne Maßnahmen, sondern nur durch integrative Ansätze gewährleistet werden.

Allerdings sollte nicht verkannt werden, dass das einkommensabhängige Elterngeld im Sinne einer nachhaltigen Familienpolitik nur einen Baustein in einem komplexeren Zusammenhang darstellen kann. Denn mit diesem Gesetz ist allenfalls ein erster Schritt sowohl hinsichtlich der finanziellen Leistungen für die Familien zur Stärkung der familiären Ressourcen wie auch hinsichtlich des Lebensverlaufs von Menschen gemacht. In der jetzigen Konstruktion wird die Zeit der Fürsorge in Abhängigkeit von der Zahl der Kinder im Rentensystem durch „Punkte“ belohnt, die aber in keiner Beziehung zum beruflichen Lebensverlauf stehen. Unter einer Lebensverlaufsperspektive und dem Grundsatz, dass gesellschaftspolitisch die Zeit für Fürsorge der beruflichen Zeit als gleichwertig gilt, ist das schwedische Modell, bei dem die Gemeinschaft der Versicherten bei einer Reduktion der Arbeitszeit bis zu bestimmten Altersgrenzen der Kinder die Sozialleistungen übernimmt, viel plausibler als das aktuelle deutsche System. Auf diese Weise entstehen Sozialversicherungs-Biografien, die bei einer vorübergehenden Teilzeittätigkeit wegen der Fürsorge für Kinder nicht geringer sind als die Sozialversicherungs-Biografien derjenigen, die keine Teilzeittätigkeit aus Fürsorgegründen ausüben. Auch lässt sich in Schweden zeigen, dass ein solches System Anreize schafft, die eigene berufliche Biografie über den Lebensverlauf so zu gestalten, dass beim Renteneintritt keine Schlechterstellung gegenüber denjenigen, die keine Fürsorge geleistet haben, besteht. Bei der Weiterentwicklung des Konzepts der nachhaltigen Familienpolitik ist auch zu fragen, ob für die Fürsorge erwachsener Kinder für die eigenen Eltern nicht ähnliche Regeln konzipiert werden sollten. Denn die Fürsorge für alt gewordene Eltern wird in einer Gesellschaft mit einer hohen Alterung zu einem wichtigen Thema der Sozialpolitik, das sich durch solche Ansätze möglicherweise angemessen stützen ließe.

Ein solches Modell nachhaltiger Familienpolitik, das die Fürsorge für die Kinder und später auch für die Eltern zum Maßstab politischen Handelns macht, muss sich auch mit der Frage auseinandersetzen, ob staatliche Leistungen für Ehe und Familie, die in keinem Zusammenhang mit den Leistungen der Personen für die Gesellschaft stehen, nicht abzuschaffen sind. Das würde die Möglichkeit schaffen, den Familien die ökonomische Existenzsicherung der Kinder über eine Grundsicherung zur Verfügung zu stellen. Auf diese Weise könnte ein Ausgleich zwischen den ökonomisch unterschiedlich leistungsfähigen Lebensformen, in denen Kinder aufwachsen, herbeigeführt werden. Heute ist eine ZweiVerdiener-Familie auch mit Kindern die vermutlich ökonomisch leistungsfähigste Le- 
bensform in Deutschland, weil die Skalenvorteile des gemeinsamen Haushalts ein effizientes Wirtschaften ermöglichen und bei zwei Einkommen auch berufliche Unsicherheiten und ökonomische Veränderungen leichter aufzufangen sind als bei nur einem Einkommen. Da aber nicht alle Kinder in dieser Lebensform aufwachsen, führt die Entscheidung der Eltern zu ihrer Lebensform häufig dazu, dass für die Kinder Hilfe zum Lebensunterhalt beantragt werden muss. In einer Gesellschaft mit pluralen Lebensformen stellt das jedoch keine angemessene Lösung für die Existenzsicherung von Kindern dar.

\section{Literatur}

Ahnert, L. \& Lamb, M. (2011). Child care and its impact on young children. In: Encyclopedia on early childhood development. http://www.child-encyclopedia.com/documents/Ahnert-LambANGxp2.pdf (Stand: 2012-09-12).

Becker, G. S. (1976). The economic approach to human behavior. Chicago: University of Chicago Press.

Belsky, R. (2012). Child care and its impacts on young children. In: Encyclopedia on early childhood development. http://www.child-encyclopedia.com/pages/PDF/child_care.pdf (Stand: 2013-09-28).

Bertram, H. (1991). Die Familie in Westdeutschland. Stabilität und Wandel familialer Lebensformen. Opladen: Leske + Budrich

Bertram, H. (1997). Familien leben. Neue Wege zur flexiblen Gestaltung von Lebenszeit, Arbeitszeit und Familienzeit. Gütersloh: Verlag Bertelsmann Stiftung.

Bertram, H. (2012). Von der skeptischen zur überforderten Generation. In: FamilienForschung BadenWürttemberg (Hrsg.), 30 Jahre FamilienForschung Baden-Württemberg. Stuttgart: FamilienForschung Baden-Württemberg, S. 91-122.

Bertram, H., Rösler, W. \& Ehlert, N. (2005). Nachhaltige Familienpolitik. Zukunftspolitik durch einen Dreiklang von Zeitpolitik, finanzieller Transferpolitik und Infrastrukturpolitik. Berlin: BMFSFJ.

Biedenkopf, K., Bertram, H. \& Niejahr, E. (2009). Starke Familie - Solidarität, Subsidiarität und kleine Lebenskreise. Bericht der Kommission „Familie und demographischer Wandel “ im Auftrag der Robert Bosch Stiftung. Renningen; F. + W. Schmidt.

Börsch-Supan, A. (2009). 50plus in Deutschland und Europa - Ergebnisse des Survey of Health, Ageing and Retirement in Europe (Alter(n) und Gesellschaft). Wiesbaden: VS Verlag für Sozialwissenschaften.

Bronfenbrenner, U. (1977). Toward an experimental ecology of human development. American Psychologist, 32, 1, S. 513-531.

Bundesministerium für Familie, Senioren, Frauen und Jugend (BMFSFJ) (Hrsg.) (1990). Achter Jugendbericht. Bericht über die Bestrebungen und Leistungen der Jugendhilfe. Bonn: BMFSFJ (Drucksache 11/6576).

Bundesministerium für Familie, Senioren, Frauen und Jugend (BMFSFJ) (Hrsg.) (1995). Fünfter Familienbericht. Familien und Familienpolitik im geeinten Deutschland-Zukunft des Humanvermögens. Bonn: BMFSFJ (Drucksache 12/7560).

Bundesministerium für Familie, Senioren, Frauen und Jugend (BMFSFJ) (Hrsg.) (2006). Siebter Familienbericht. Familie zwischen Flexibilität und Verlässlichkeit. Perspektiven für eine lebenslaufbezogene Familienpolitik. Berlin: BMFSFJ (Drucksache 16/1360).

Bundesministerium für Familie, Senioren, Frauen und Jugend (BMFSFJ) (Hrsg.) (2009). Familienreport 2009. Leistungen, Wirkungen, Trends. Berlin: BMFSFJ.

Bundesministerium für Familie, Senioren, Frauen und Jugend (Hrsg.) (2010). Ausgewählte Aspekte der Wirkung lokaler Bündnisse für Familie. Evaluation von Ramboll Management Consulting und DIW. Berlin: econ.

Bundesministerium für Familie, Senioren, Frauen und Jugend (Hrsg.) (2013). Bestandsaufnahme der familienbezogenen Leistungen und Maßnahmen des Staates im Jahr 2010. http://www.bmfsfj.de/ 
RedaktionBMFSFJ/Abteilung2/Pdf-Anlagen/familienbezogene-leistungen-tableau-2010,property $=$ pdf, bereich $=$ bmfsfj, sprache $=$ de, $r w b=$ true.pdf (Stand: 2013-05.27).

Bundesministerium für Jugend, Familie und Gesundheit (BJFG) (1975). Zweiter Familienbericht. Familie und Sozialisation. Leistungen und Leistungsgrenzen der Familie hinsichtlich der Erziehungsund Bildungsprozesse der jungen Generation. Bonn: BJFG (Drucksache 7/3502).

Bundesverfassungsgericht (BVerfG) (2010). 1 BvL 1/09 vom 9.2.2010, Absatz-Nr. (1-220). http://www. bverfg.de/entscheidungen/1s20100209_1bv1000109.html; (Stand: 2012-09-25).

Coleman, J. S. (1979). Equality of educational opportunity. New York: Arno Press.

Erler, G., Jaeckel, M. \& Sass, J. (1983). Mutter zwischen Beruf und Familie: Familienpolitik mit Mutterschaftsurlaub, Elternurlaub oder Erziehungsgeld? Was Frauen davon halten. Modelle und Meinungen aus fünf europäischen Ländern. München: Juventa Verlag: München (Reihe Deutsches Jugendinstitut).

Habisch, A. (1999). Sozialpolitik als Gesellschaftsordnungspolitik - Wilfrid Schreiber als Mitarchitekt der sozialen Marktwirtschaft. Ingolstadt: Katholische Universität Eichstätt (Diskussionsbeiträge der Wirtschaftswissenschaftlichen Fakultät Ingolstadt).

Hermann, C. (1984). Gleichstellung der Frau und Rentenrecht. Zur bevorstehenden Reform der Alterssicherung. Berlin: Duncker \& Humblot.

Hoem, B. \& Hoem, J. M. (1996). Sweden's family policies and rollercoaster fertility. Journal of Population Problems, 52, 1, S. 1-22.

Kohli, M., Hank, K. \& Künemund, H. (2009). The social connectedness of older Europeans: Patterns, dynamics and contexts. Journal of European Social Policy 19, 1, S. 327-340.

Kommission der Europäischen Gemeinschaften (2005), Grünbuch „Angesichts des demografischen Wandels - eine neue Solidarität zwischen den Generationen “. Brüssel: Kommission der Europäischen Gemeinschaften.

Michel, S. \& Mahon, R. (2002). Child care policy at the crossroads. Gender and welfare state restructuring. Routledge: New York.

Sachße, C. (1996). Recht auf Erziehung - Erziehung durch Recht. Entstehung, Entwicklung und Perspektiven des Jugendhilferechts. Zeitschrift für Sozialreform, 42, 9, S. 557-571.

Schreiber, W. (2004). Existenzsicherheit in der industriellen Gesellschaft. Köln: In: Bund katholischer Unternehmer (BKU) (Hrsg.), Diskussionsbeiträge, 28, S. 1-50.

Shonkoff, J. P. \& Phillips, D. A. (Hrsg.) (2000). From neurons to neighborhoods. The science of early childhood development. Washington, DC: National Academies Press.

Statistisches Bundesamt (Hrsg.) (2011). Statistiken der Kinder- und Jugendhilfe. Kinder und tätige Personen in Tageseinrichtungen und in öffentlich geförderter Kindertagespflege am 01.03.2011. Wiesbaden: Statistisches Bundesamt.

Waldfogel, J. (2006). What children need. Cambridge: Harvard University Press.

Waldfogel, J., Craigie, T.-A. \& Brooks-Gunn, J. (2010). Fragile families and child wellbeing. The Future of Children, 20, 2, S. 87-112.

Wissenschaftlicher Beirat für Familienfragen (Hrsg.) (1984). Gutachten „Familie und Arbeitswelt“. Stuttgart: Kohlhammer (Schriftenreihe des Bundesministers für Jugend, Familie und Gesundheit, Band 143).

Wolf, K. \& Grgic, M. (2009). Kinderbetreuung im europäischen Vergleich. Die Chancen von EU-SILC und die aktuellen Grenzen. München: Deutsches Jugendinstitut (Wissenschaftliche Texte).

Eingereicht am/Submitted on: 31.10.2012

Angenommen am/Accepted on: 03.07.2013 
Anschrift des Autors und der Koautorin/Addresses of author and co-author:

Prof. Dr. Hans Bertram (Korrespondenzautor/Corresponding author)

Carolin Deuflhard, B.A.

Humboldt-Universität zu Berlin

Institut für Sozialwissenschaften

Mikrosoziologie

Unter den Linden 6

10099 Berlin

Deutschland/Germany

E-Mail: hbertram@rz.hu-berlin.de deuflhac@cms.hu-berlin.de 